\title{
Impacto de la pandemia por COVID-19 en un programa de cirugía cardiovascular pediátrica de un hospital público de Argentina
}

\author{
Impact of the COVID-19 pandemic on a pediatric cardiovascular surgery \\ program of a public hospital from Argentina
}

\author{
Dr. Ricardo Rodríguez ${ }^{a}$, Dra. María Althabe ${ }^{a}$, Dra. Mariela Krynskia , Dra. Mercedes Montonati ${ }^{a}$, \\ Dra. María L. Pilan ${ }^{a}$, Dra. Belén Desocio ${ }^{a}$, Dr. Guillermo Moreno ${ }^{a}$, Dra. Gladys Salgado ${ }^{b}$ Dr. Javier Cornelis ${ }^{c}$ y \\ Dra. Miriam Lenz ${ }^{a}$
}

\section{RESUMEN}

Objetivo. Describir el impacto de la pandemia por COVID-19 en el programa de cirugía cardiovascular pediátrica y estimar el tiempo para reducir la lista de espera quirúrgica.

Métodos. Estudio descriptivo y retrospectivo. Se compararon resultados quirúrgicos del período preCOVID versus el período COVID.Seutilizóun modelo matemático para estimar el tiempo para reducir la lista de espera.

Resultados. Entre el 23 de marzo y el 31 de agosto de 2020 se operaron 83 pacientes, que representan una reducción del $60 \%$, respecto al período preCOVID. La mediana de edad fue de 6 meses (rango intercuartílico [RIC]: 25-75, 1,8 meses a 2,9 años; $p=0,0023$. El tiempo para eliminar la lista de espera varía entre 10 y 19 meses.

Conclusiones. El programa tuvo una reducción del $60 \%$. El tiempo de resolución de la lista de espera puede ser al menos 10 a 19 meses.

Palabras clave: COVID-19; procedimientos quirúrgicos cardíacos; cardiopatías congénitas.

http: / / dx.doi.org/10.5546/ aap.2021.266

Texto completo en inglés:

http: / / dx.doi.org/10.5546/ aap.2021.eng.266

Cómo citar: Rodríguez R, Althabe M, Krynski M, Montonati M, et al. Impacto de la pandemia por COVID-19 en un programa de cirugía cardiovascular pediátrica de un hospital público de Argentina. Arch Argent Pediatr 2021;119(4):266-270.

a. Servicio de Terapia Intensiva (UCI 35).

b. Servicio de Cardiología.

c. Servicio de Cirugía Cardiovascular.

Hospital de Pediatría Dr. Prof. Juan P. Garrahan, Ciudad

Autónoma de Buenos Aires, Argentina.

Correspondencia:

Dr. Ricardo Rodríguez: ricardo.rodriguez75@gmail.com

Financiamiento: Ninguno.

Conflicto de intereses: Ninguno que declarar.

Recibido: 30-9-2020

Aceptado: 23-2-2021

\section{INTRODUCCIÓN}

Desde el inicio de la pandemia por COVID-19 iniciada en Wuhan y luego extendida a todo el mundo, los diferentes servicios médicos tuvieron que adaptar su estructura funcional de acuerdo con las necesidades de cada país. El Hospital de Pediatría Dr. Prof. Juan P. Garrahan es un centro de referencia de alta complejidad de la región, en el que se realizan 750 cirugías por año en pacientes con cardiopatías congénitas.

Nuestro país implementó una de las cuarentenas más prolongadas, todos los procedimientos diagnósticos y terapéuticos diferibles fueron reprogramados. ${ }^{1}$ El alto costo de las listas de espera en cirugía de cardiopatía congénita es conocido ${ }^{2,3}$ y no solo afecta al número de cirugías, sino también a los estudios diagnósticos y los procedimientos intervencionistas.

El objetivo del estudio fue describir el impacto de la pandemia por COVID-19 en el programa de cirugía cardiovascular del Hospital de Pediatría Dr. Prof. Juan P. Garrahan, y estimar el tiempo para reducir la lista de espera con diferentes escenarios de aumento de la oferta de turnos quirúrgicos y/o reducción transitoria de la demanda de pacientes.

\section{POBLACIÓN Y MÉTODOS}

Estudio retrospectivo y descriptivo. Se incluyeron pacientes operados y recuperados en la unidad de recuperación cardiovascular del Hospital Garrahan desde el 23 de marzo al 31 de agosto de 2020 (período COVID) y se compararon con el promedio de igual período de los últimos dos años (período preCOVID). Se analizó el número de pacientes operados, la edad, la complejidad del procedimiento por el ajuste de riesgo para cirugía por cardiopatía congénita (RACHS-1, por su sigla en inglés) ${ }^{4}$ la procedencia, los días de internación, el porcentaje 
de pacientes en oxigenación por membrana extracorpórea (ECMO, por su sigla en inglés) y la mortalidad posoperatoria a 30 días. El estudio fue aprobado por la Dirección Asociada de Docencia e Investigación del Hospital, quienes no consideraron necesario la solicitud de consentimiento. Los resultados se expresaron como mediana y rango intercuartílico (RIC 25-75) o valor absoluto y porcentaje, odds ratio (OR) e intrevalo de confianza del $95 \%$ (IC95\%), según correspondiera. La comparación se realizó con la prueba de chi cuadrado para las variables discretas y la prueba de Wilcoxon (u-test) para las continuas, considerando como significativa una $p<0,05$. Se utilizó el paquete estadístico Stata $12^{\circledR}$.

Para estimar el tiempo necesario para recuperar la lista de espera generada por los casos no operados durante la pandemia, se utilizó la fórmula propuesta por Salenger R y col. ${ }^{5}$

\section{Casos no operados (CNO)}

(cirugias preCOVID - cirugias en COVID) $x$ (días de restricción ${ }^{*}$ )

\footnotetext{
* Los días de restricción se cuentan cómo días hábiles desde el inicio de las medidas hasta la fecha en la que se retoma la capacidad operativa completa.
}

\section{Tiempo de resolución de la lista de espera (TR)}

(1 - cirugías en COVID/cirugias preCOVID) $x$

días de restricción $\left.n^{*}\right) /\left(a^{*}-1\right)$

* El factor de aceleración (a) representa el aumento de turnos por sobre la línea de base del programa.
La capacidad operativa es de 13 turnos quirúrgicos por semana, excepto en período de vacaciones, que se reducen a 10 turnos.

Se calcularon los días necesarios para recuperar los $\mathrm{CNO}$, asumiendo que la demanda habitual del programa se va a sostener una vez superada la pandemia. Se analizaron tres escenarios diferentes de incrementos: $15 \%, 20 \%$ y $30 \%$ con la misma demanda. Se estudiaron también otros tres escenarios en los que se suma una reducción estimada de la demanda de $15 \%$ por pacientes que pudieron haber perdido su oportunidad quirúrgica, se operaron o pudieron haberse operado en otra institución, y aquellos que pueden retrasar su reparación quirúrgica por más de dos años sin riesgo clínico.

\section{RESULTADOS}

En el período COVID se operaron 83 pacientes, mientras que en el período preCOVID se operaron 237. Esto representa una reducción del $60 \%$ en relación al promedio de los dos años anteriores en el mismo periodo (Figura 1). La mediana de edad de los pacientes en el período COVID fue de 6 meses (RIC: 25-75, 1,8 m-2,9 a) versus período preCOVID, con una mediana de edad de 16 m (RIC: 3 m-6,2 a); $\mathrm{p}=0,0023$. $\mathrm{Al} 14 \%$ de los pacientes se les realizó una cirugía de alta complejidad medida con puntuación de RACHS $\geq 4$. Los resultados de la cirugía fueron similares en los dos períodos.

Las variables analizadas y su significación estadística se describen en la Tabla 1. Por la

TABLA 1. Características de la población comparando los períodos preCOVID y COVID

\begin{tabular}{|c|c|c|c|c|}
\hline & $\begin{array}{l}\text { Período preCOVID } \\
\quad(\mathbf{N}=\mathbf{2 3 7})\end{array}$ & $\begin{array}{l}\text { Período COVID } \\
\quad(\mathbf{N}=\mathbf{8 3})\end{array}$ & Valor de $p$ & OR (IC95\%) \\
\hline Cirugías con CEC & $210(88,6 \%)$ & $75(90,3 \%)$ & 0,98 & $1,01(0,70-1,46)$ \\
\hline Edad (mediana, RIC 25-75) & $16 \mathrm{~m}(3 \mathrm{~m}-6,2 \mathrm{a})$ & $6 \mathrm{~m}(1,8 \mathrm{~m}-2,9 \mathrm{a})$ & 0,0023 & ND \\
\hline $\begin{array}{l}\text { Días de internación } \\
\text { (mediana, RIC 25-75) }\end{array}$ & $5,5(3-11)$ & $6(4-15)$ & 0,65 & ND \\
\hline Recién nacidos & $28(11,8 \%)$ & $14(16,8 \%)$ & 0,40 & $1,42(0,71-2,84)$ \\
\hline Diagnóstico prenatal & $14(7,9 \%)$ & $4(8 \%)$ & 0,94 & $0,81(0,26-2,54)$ \\
\hline Procedencia (AMBA) & $119(50,2 \%)$ & $48(57,8 \%)$ & 0,57 & $1,15(0,75-1,74)$ \\
\hline Puntaje de RACHS- $1 \geq 4$ & $24(10,1 \%)$ & $11(13,2 \%)$ & 0,61 & $1,30(0,61-2,78)$ \\
\hline ECMO & $6(2,5 \%)$ & $3(3,6 \%)$ & 0,90 & $1,42(0,34-5,83)$ \\
\hline Mortalidad POP & $8 \%$ & $8,7 \%$ & 0,81 & $0,77(0,28-2,15)$ \\
\hline Trasplantes cardíacos & 3 pacientes & 4 pacientes & & \\
\hline
\end{tabular}

CEC: circulación extracorpórea, RIC: rango intercuartílico, RACHS-1: ajuste de riesgo para cirugía por cardiopatía congénita, por su sigla en inglés), AMBA: Área Metropolitana de Buenos Aires, ECMO: oxigenación de membrana extracorpórea, POP: posoperatoria, IC95\%: intervalo de confianza del $95 \%$, OR: odds ratio. 
reducción de turnos quirúrgicos en los 102 días hábiles analizados, se dejaron de operar 183 pacientes. El tiempo necesario para eliminar esta lista de espera, una vez incrementada la capacidad operativa, varía entre 10 y 19 meses, según los diferentes escenarios analizados (Figuras 2 y 3 ).

\section{DISCUSIÓN}

El programa de cirugía cardiaca sufrió una reducción importante desde el inicio de la pandemia. En nuestro país, cada año, aproximadamente 4300 niños requieren una cirugía cardiaca en los primeros años de vida. ${ }^{6,7}$ Con el objetivo de mantener la continuidad de las cirugías durante este período de pandemia,

FIGURA 1. Número de pacientes operados por mes: comparación de los períodos preCOVID (2018-2019) y COVID (2020)

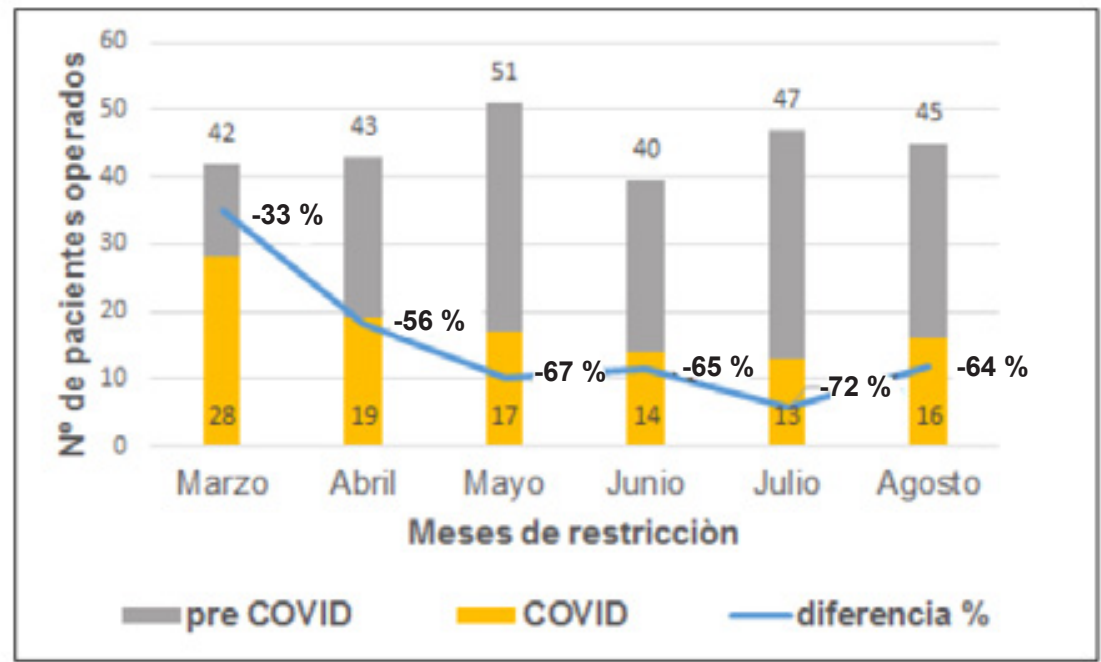

Nota: la línea marca la reducción porcentual mensual, el promedio de la reducción en el periodo es del $60 \%$.

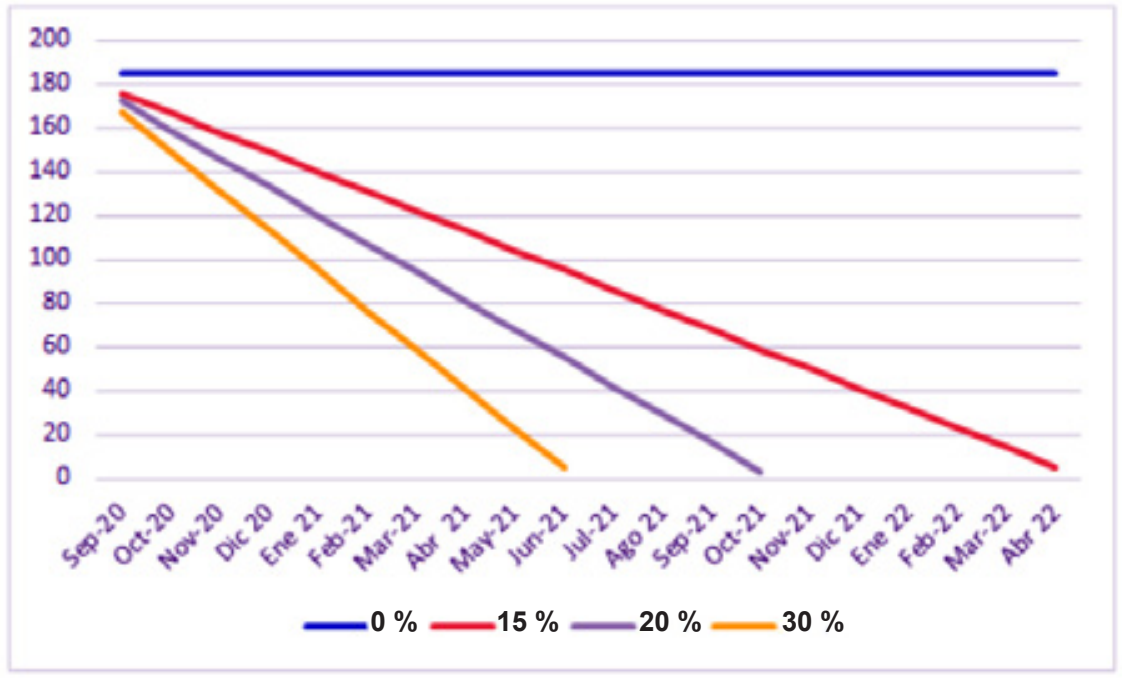

Nota: para esta estimación, se consideró el total de los casos no operados (183) en el período COVID. 
se implementaron estrategias de protección para el personal y los pacientes; entre ellas, la cohortización de equipos de trabajo y reducción de la actividad programada para liberar camas de terapia intensiva para pacientes con COVID. A pesar de todos los esfuerzos realizados, la cantidad de pacientes operados se vio reducida en forma significativa, y probablemente la capacidad operativa habitual no se recupere por unos meses. Durante este período se priorizaron: a) pacientes con indicación de cirugía inmediata: recién nacidos, pacientes con cianosis grave, insuficiencia cardíaca; b) pacientes cuya falta de resolución en tiempo oportuno los expondría a un riesgo aumentado; ${ }^{8}$ y c) pacientes en lista de espera de trasplante cardíaco. Se suspendieron todas las cirugías de pacientes cuya evolución no se afectaría de manera significativa por la demora quirúrgica. Con estas consideraciones, nuestro programa sufrió una reducción inicial del $60 \%$ durante el período evaluado en relación con el volumen operado en igual período durante la etapa preCOVID. Estos resultados son similares a los de otros centros como citan Polo López y col., ${ }^{9}$ que describen una reducción de $51 \%$ menos de cirugías mayores y $54 \%$ menos de cirugías extracorpóreas. Protopapas y col., ${ }^{10}$ realizaron una encuesta mundial y encontraron que $51 \%$ de los servicios de cirugía cardiovascular consultados disminuyeron más de $50 \%$ su producción.

Los días de restricción en nuestro estudio se contaron como días hábiles desde el inicio de las medidas hasta el 31 de agosto de 2020, fecha en la que se retoma parte de la capacidad operativa.
Si bien la estimación del tiempo que demandará recuperar los pacientes no operados es sencilla de estimar, la implementación de estrategias para minimizar el impacto de la demora quirúrgica en pacientes no operados podría ser algo más compleja.

El modelo que utilizamos para estimar la magnitud del problema tiene limitaciones, pero resulta una aproximación útil para analizar diferentes escenarios. El estudio multicéntrico publicado por Protopapas y col., dice que el intervalo de tiempo estimado necesario para recuperar el funcionamiento normal de las restricciones pospandémicas fue de uno a dos meses en el $43 \%$ de los programas y de dos a cuatro meses en el $31 \%$. Dado que nuestra capacidad operativa ya se encontraba al límite antes de la pandemia, la posibilidad de aumentar el número de turnos quirúrgicos más del 15-20 \% es muy improbable. Nuestro modelo arroja así una estimación al menos de 10 a 19 meses para reducir de manera significativa esta nueva lista de espera. Salenger y col., ${ }^{5}$ describen una caída del $54 \%$ en el volumen quirúrgico cardíaco en pacientes adultos; este retraso requeriría un volumen operativo mensual del $216 \%$ al $263 \%$ del valor inicial; en vista de que sería un desafío lograr esto en un mes, predijeron que la cantidad de tiempo necesaria para despejar el atraso variaría de 1 a 8 meses.

A los problemas de disponibilidad de turnos quirúrgicos se le deben sumar otros potenciales cuellos de botella como la disponibilidad de camas de terapia intensiva, camas de sala y

FIGURA3. Tiempo de resolución de la lista de espera considerando escenarios de incremento de la oferta y disminución de la demanda

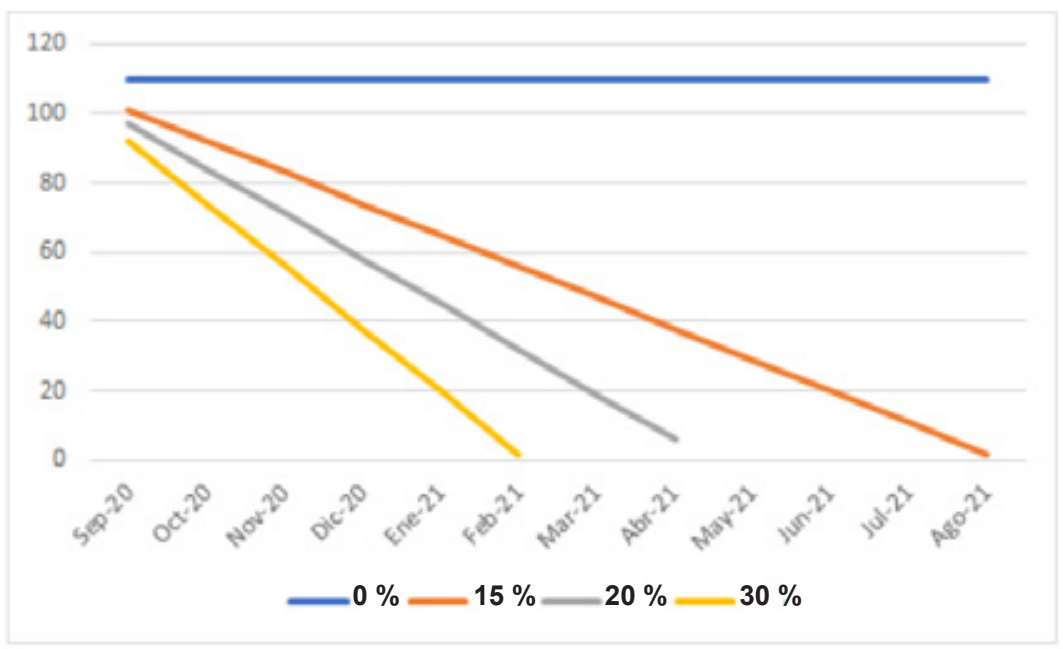

Nota: en estos escenarios, al incremento de turnos se le agregó una reducción estimada del $15 \%$ de la demanda por diferentes causas. 
turnos para estudios complementarios, como cateterismos, estudios electrofisiológicos, imágenes, entre otros.

Si bien el objetivo del trabajo es mostrar un aspecto de los efectos de la pandemia, probablemente se requerirá esfuerzos extraordinarios para recuperar la actividad en relación con la era preCOVID.

Las limitaciones se relacionan con un estudio retrospectivo y con la aplicación de un modelo matemático en el que solo se hace una estimación teórica de los pacientes no operados, y no se evaluaron otros riesgos como oportunidad quirúrgica perdida en grupos de pacientes de mayor riesgo, seguimiento y estudios no realizados.

\section{CONCLUSIONES}

El programa de cirugía cardiovascular tuvo una reducción del $60 \%$ de su capacidad operativa entre los meses de marzo a agosto en comparación con un período similar preCOVID. El tiempo de resolución de la lista de espera generada por la pandemia puede ser, al menos, 10 a 19 meses, aun con la implementación de estrategias de aumento de la oferta y postergando la resolución de pacientes cuya condición permite diferir la cirugía.

\section{REFERENCIAS}

1. HospitalGarrahan. Licencias extraordinarias en el marco de las medidas adoptadas por el gobierno nacional. Intranet, noticias internas. 2020.

2. Althabe M, Rodríguez R, Balestrini M, Charroqui A, et al. Morbilidad en cirugía de cardiopatía congénita en un hospital público en Argentina. Arch Argent Pediatr. 2018; 116(1):e14-8.

3. Magliola R, Laura JP, Capelli H. Situación actual de los niños con cardiopatía congénita en Argentina. Arch Argent Pediatr. 2000; 98(2):130-3.

4. Jenkins KJ. Risk adjustment for congenital heart surgery: the RACHS-1 method. Semin Thorac Cardiovasc Surg Pediatr Card Surg Anпu. 2004; 7(1):180-4.

5. Salenger R, Etchill EW, Ad N, Matthew T, et al The Surge after the Surge: Cardiac Surgery post-COVID-19. Ann Thorac Surg. 2020; 110(6):2020-5.

6. Villa A, Olivetti E, Capelli H. Programa Nacional de Cardiopatías Congénitas: diseño, implementación y resultados a mediano plazo. Rev Argent Salud Publica. 2015; 6(24):39-42.

7. Magliola RH, Althabe M, Moreno G, Lenz A, et al. Cardiopatías congénitas: Resultados quirúrgicos en un hospital público en Argentina. Arch Cardiol Mex. 2011; 81(3):178-82.

8. Stephens EH, Dearani JA, Guleserian KJ, Overman D, et al. COVID-19: Crisis Management in Congenital Heart Surgery. Ann Thorac Surg. 2020; 110(2):701-6.

9. Polo López L, Centella Hernández T, González Calle A, Bautista Hernández V, et al. Cirugía de cardiopatías congénitas en España durante el estado de alarma por COVID-19. Cir Cardiovasc. 2020; 27(4):137-41.

10. Protopapas E, Lo Rito M, Vida V, Sarris G, et al. Early Impact of the COVID-19 Pandemic on Congenital Heart Surgery Programs Across the World: Assessment by a Global Multi-Societal Consortium. World J Pediatr Congenit Heart Surg. 2020; 11(6):689-96.

\title{
Simulación de intubación pediátrica con videolaringoscopio de bajo costo en contexto de pandemia por COVID-19
}

\author{
Simulation of pediatric intubation using a low-cost videolaryngoscope in the \\ setting of the COVID-19 pandemic
}

\author{
Dr. Daniel Rufach ${ }^{a}$ Dra. Silvia Santos ${ }^{b}$ y Dr. Marcelo Terebiznik ${ }^{a}$
}

a. Hospital Interzonal de Agudos "Eva Perón”, San Martín, provincia de Buenos Aires, Argentina.

b. Hospital General de Pediatría "Prof. Dr. Juan P. Garrahan", Ciudad Autónoma de Buenos Aires, Argentina.

Correspondencia:

Dr. Daniel Rufach: jrufach@intramed.net

Financiamiento: Ninguno.

Conflicto de intereses: Ninguno que declarar.

Recibido: 13-9-2020

Aceptado: 1-2-2021

\begin{abstract}
RESUMEN
En pacientes con infección por SARS-CoV-2 la intubación endotraqueal es un procedimiento con riesgo elevado de contagio. La videolaringoscopia complementa la protección del profesional, pero los videolaringoscopios comerciales son caros y no siempre están disponibles en las terapias intensivas pediátricas argentinas. El objetivo fue describir la práctica de intubación en un modelo de cabeza de simulación de lactante con un videolaringoscopio artesanal de bajo costo.

Quince pediatras sin experiencia previa con el dispositivo participaron de una práctica de intubación en una cabeza de simulación con un videolaringoscopio artesanal. El tiempo promedio del primer intento fue de 116,4 segundos (intervalo de confianza del $95 \%$ [IC95\%]: 84,8-148,0) y, el del siguiente
\end{abstract}

